\title{
On the basic set of solutions of a high-order linear difference equation.
}

\author{
F. Marcellán \\ Departamento de Matemáticas \\ Universidad Carlos III de Madrid \\ Avenida Universidad 30 \\ 28911 Leganés, Spain. \\ S.M. Zagorodnyuk \\ School of Mathematics and Mechanics \\ Karazin Kharkiv National University \\ Kharkiv, 61077, Ukraine.
}

Abstract. In this paper we analyze the set of solutions of a linear difference equation of high-order. More precisely, we obtain a basis of such a linear subspace. We deduce integral representations for elements of this basis. ${ }^{1} 2$

\section{$\S 1$. Introduction.}

Linear difference equations appear in the framework of the theory of orthogonal polynomials in a natural way $[\mathrm{C}]$. Namely, consider a linear functional $u$ defined in the linear space $\mathbb{P}$ of polynomials with complex coefficients.

Let denote $u_{n}=<u, x^{n}>$ the moment of order $n$ associated with the linear functional $u$.

The linear functional $u$ is said to be quasi-definite if the Hankel matrices $H_{n}=\left[u_{j+k}\right]_{j, k=0}^{n}, n=0,1,2, \ldots$ are nonsingular for every $n=0,1,2, \ldots$ In such a situation there exists a sequence of monic polynomials $\left\{P_{n}\right\}_{n=0}^{\infty}$ such that

(i) $\operatorname{deg} P_{n}=n$,

(ii) $<u, P_{n} P_{m}>=k_{n} \delta_{n, m}$, with $k_{n} \neq 0$.

$\left\{P_{n}\right\}_{n=0}^{\infty}$ is said to be the sequence of monic polynomials orthogonal with respect to $u$. For a sake of simplicity we will write SMOP.

\footnotetext{
${ }^{1}$ Mathematical Subject Classification 2000: 42C05

${ }^{2}$ Key words: high-order linear difference equations, orthogonal polynomials, matrix of measures
} 
It is very well known that the $\operatorname{SMOP}\left\{P_{n}\right\}_{0}^{\infty}$ satisfies a three-term recurrence relation

$$
x P_{n}(x)=P_{n+1}(x)+\beta_{n} P_{n}(x)+\gamma_{n} P_{n-1}(x), n \geq 1,
$$

where $\gamma_{n} \neq 0, n=1,2, \ldots$

In other words, $\left\{P_{n}\right\}_{n=0}^{\infty}$ is a solution of the linear difference equation

$$
x y_{n}=y_{n+1}+\beta_{n} y_{n}+\gamma_{n} y_{n-1}, n \geq 1,
$$

with initial conditions $y_{0}=1$ and $y_{1}=x-\beta_{0}$, or, equivalently, $y_{-1}=0, y_{0}=1$ if (1.1) is valid for $n \geq 0$. Taking into account the set of solutions of (1.1) is a two dimensional linear space, a second linearly independent solution of (1.1) is the sequence $\left\{R_{n}\right\}_{n=-1}^{\infty}$ associated with initial conditions $y_{-1}=1, y_{0}=0$ $\left(\gamma_{0}=-1\right)$. Notice that in such a case, $R_{n}$ is a polynomial of degree $n-1$, where $n \geq 1$. Denote $P_{n}^{(1)}(x)=R_{n+1}(x), n=0,1, \ldots$. We will say $\left\{P_{n}^{(1)}\right\}_{0}^{\infty}$ is the sequence of associated polynomials of the first kind. (Also, polynomials $\left\{P_{n}(x)\right\}_{0}^{\infty},\left\{P_{n}^{(1)}(x)\right\}_{0}^{\infty}$ are often called the polynomials of the first kind and the polynomials of the second kind respectively $[\mathrm{A}])$. Thus, $\left\{P_{n}^{(1)}\right\}_{0}^{\infty}$ satisfies a three-term recurrence relation

$$
x P_{n}^{(1)}(x)=P_{n+1}^{(1)}(x)+\beta_{n+1} P_{n}^{(1)}(x)+\gamma_{n+1} P_{n-1}^{(1)}(x), n \geq 1,
$$

with initial conditions $P_{0}^{(1)}(x)=1$ and $P_{1}^{(1)}(x)=x-\beta_{1}$. Taking into account $\gamma_{n+1} \neq 0$ and according to the Favard's theorem (see [C]) there exists a quasidefinite linear functional $u^{(1)}$ such that $\left\{P_{n}^{(1)}\right\}_{0}^{\infty}$ is the corresponding SMOP.

It is very easy to check that

$$
P_{n}^{(1)}(x)=\frac{1}{u_{0}}<u, \frac{P_{n+1}(x)-P_{n+1}(u)}{x-u}>.
$$

On the other hand, every solution of (1.1) can be given as

$$
y_{n}=A(x) P_{n}(x)+B(x) P_{n-1}^{(1)}(x),
$$

where $A$ and $B$ are functions which can be explicitly given in terms of the initial conditions for (1.1).

Assuming $u$ is a quasi-definite linear functional, an indefinite inner product can be defined as

$$
(p, q)=<u, p q>, p, q \in \mathbb{P}
$$


Thus $(x p, q)=(p, x q)$, i.e. the multiplication by $x$ is a symmetric operator in $\mathbb{P}$ with respect to $($,$) . Indeed the three-term recurrence relation is a$ straightforward consequence of this fact. Favard's theorem can be read in this context as follows: There exists an indefinite inner product (1.2) such that the multiplication by $x$ is a symmetric operator with respect to (1.2) and this inner product has an integral representation.

A natural extension of this problem is the following: To characterize inner products in $\mathbb{P}$ such that the multiplication by a polynomial $h$ is a symmetric operator with respect to the inner product ([DA], $[\mathrm{MSz}])$.

For instance, if $h(x)=x^{N}, N \geq 2$, then an inner product satisfying the above condition is (1.2) as well as another example is

$$
(p, q)=<u, p q>+\sum_{k=1}^{N-1} M_{k} p^{(k)}(0) \overline{q^{(k)}(0)} .
$$

In [D1] the set of all inner products such that $h(x)=x^{N}$ is a symmetric operator is characterized as well as some extra conditions about the operator in order the inner product be of the form (1.3) are given. In [Z1], [Z2], [Z3] the case $N=2$ is considered and in [Z4], [Z5] the case of arbitrary $N$ is studied.

If we denote $\left\{P_{n}\right\}_{0}^{\infty}$ the sequence of monic polynomials orthogonal with respect to an indefinite inner product $($,$) , i.e.$

(i) $\operatorname{deg} P_{n}=n$,

(ii) $\left(P_{n}, P_{m}\right)=k_{n}, \delta_{n, m}, k_{n} \neq 0$,

then the symmetry of $x^{N}$ with respect to $($,$) reads$

$$
x^{N} P_{n}(x)=\sum_{k=-N}^{N} \alpha_{n, k} P_{n+k}(x),
$$

where

$$
\begin{gathered}
\alpha_{n, k}=\frac{\left(x^{N} P_{n}(x), P_{n+k}(x)\right)}{\left(P_{n+k}(x), P_{n+k}(x)\right)}=\frac{\left(P_{n}(x), x^{N} P_{n+k}(x)\right)}{\left(P_{n+k}(x), P_{n+k}(x)\right)}= \\
=\overline{\alpha_{n+k, n}} \frac{\left(P_{n}(x), P_{n}(x)\right)}{\left(P_{n+k}(x), P_{n+k}(x)\right)} \\
\quad \text { or } k= \pm 1, \pm 2, \ldots, \pm N, \text { and } \\
\alpha_{n, N}=1, \alpha_{n,-N}=\frac{\left(P_{n}(x), P_{n}(x)\right)}{\left(P_{n-N}(x), P_{n-N}(x)\right)} \neq 0 .
\end{gathered}
$$


If we assume the inner product is positive definite, then a sequence of orthonormal polynomials can be introduced

$$
p_{n}(x)=\left(P_{n}(x), P_{n}(x)\right)^{-\frac{1}{2}} P_{n}(x) .
$$

Thus (1.4) becomes

$$
x^{N} p_{n}(x)=\beta_{n, 0} p_{n}(x)+\sum_{k=1}^{N}\left(\overline{\beta_{n-k, k}} p_{n-k}(x)+\beta_{n, k} p_{n+k}(x)\right),
$$

with

$$
\beta_{n, k}=\alpha_{n, k}\left[\frac{\left(P_{n+k}(x), P_{n+k}(x)\right)}{\left(P_{n}(x), P_{n}(x)\right)}\right]^{\frac{1}{2}}, k= \pm 1, \pm 2, \ldots, \pm N .
$$

The aim of our contribution is twofold. First, to describe a basis of the linear subspace of solutions for the higher order linear difference equation

$$
x^{N} y_{n}=\beta_{n, 0} y_{n}+\sum_{k=1}^{N}\left(\overline{\beta_{n-k, k}} y_{n-k}+\beta_{n, k} y_{n+k}\right) .
$$

Thus an extension of the associated polynomials of the first kind appears in a natural way. For $N=2$, see [Z1] and [Z2].

Second, we use the extensions of the Favard's theorem ([D2], [Z4], [Z5]) to describe the corresponding inner products associated with the elements of the basis obtained above and to deduce integral representations for associated polynomials.

\section{$\S 2$. Basic solutions.}

Let us consider the following $(2 N)$-order linear difference equation:

$$
\begin{gathered}
\sum_{i=1}^{N}\left(\overline{\alpha_{k-i, i}} y_{k-i}+\alpha_{k, i} y_{k+i}\right)+\alpha_{k, 0} y_{k}=\lambda^{N} y_{k}, k=N, N+1, N+2, \ldots ; \\
\alpha_{m, n} \in \mathbb{C}, m \in \mathbb{Z}_{+}, n=0,1,2, \ldots, N: \alpha_{m, N} \neq 0, \alpha_{m, 0} \in \mathbb{R} ; N \in \mathbb{N} ; \\
\lambda \in \mathbb{C} \text { is a parameter and } y=\left(\begin{array}{c}
y_{0} \\
y_{1} \\
y_{2} \\
\cdot \\
\cdot
\end{array}\right) \text { is a vector solution; }
\end{gathered}
$$


We assume the initial conditions

$$
\left(y_{0}, y_{1}, \ldots, y_{2 N-1}\right)^{T}=\left(y_{0}^{0}, y_{1}^{0}, \ldots, y_{2 N-1}^{0}\right)^{T}, y_{i}^{0} \in \mathbb{C}, i=0,1, \ldots, 2 N-1
$$

Let $\left\{p_{n}(\lambda)\right\}_{n=0}^{\infty}$ be a system of polynomials ( $p_{n}$ is of the $n$-th degree) such that:

$$
\begin{gathered}
\left(\begin{array}{cccccccc}
\alpha_{0,0} & \alpha_{0,1} & \alpha_{0,2} & \ldots & \alpha_{0, N} & 0 & 0 & \ldots \\
\overline{\alpha_{0,1}} & \alpha_{1,0} & \alpha_{1,1} & \ldots & \alpha_{1, N-1} & \alpha_{1, N} & 0 & \ldots \\
\vdots & \vdots & \vdots & \ddots & \vdots & \vdots & \vdots & \ldots \\
\overline{\alpha_{0, N}} & \frac{\alpha_{1, N-1}}{\alpha_{2, N-2}} & \ldots & . & . & . & \ldots \\
0 & \overline{\alpha_{1, N}} & \overline{\alpha_{2, N-1}} & \ldots & . & . & . & \ldots \\
\vdots & \vdots & \vdots & \vdots & \vdots & \vdots & \vdots & \ddots
\end{array}\right)\left(\begin{array}{c}
p_{0}(\lambda) \\
p_{1}(\lambda) \\
\vdots \\
p_{N}(\lambda) \\
p_{N+1}(\lambda) \\
\vdots
\end{array}\right)= \\
=\lambda^{N}\left(\begin{array}{c}
p_{0}(\lambda) \\
p_{1}(\lambda) \\
\vdots \\
p_{N}(\lambda) \\
p_{N+1}(\lambda) \\
\vdots
\end{array}\right)
\end{gathered}
$$

So, $\left\{p_{n}(\lambda)\right\}_{0}^{\infty}$ is a solution of $(2.1)$ which additionally satisfies the relations of $(2.1)$ for $k=0,1,2, \ldots, N-1 ; p_{-k}=0, k=1,2, \ldots, N$. We put by definition

$$
\begin{gathered}
p\left(u_{1} ; u_{2}\right)=\frac{p\left(u_{1}\right)-p\left(u_{2}\right)}{u_{1}-u_{2}}, \\
p\left(u_{1} ; u_{2} ; u_{3} ; \ldots ; u_{k}\right)=\frac{p\left(u_{1} ; u_{3} ; u_{4} ; \ldots ; u_{k}\right)-p\left(u_{2} ; u_{3} ; u_{4} ; \ldots ; u_{k}\right)}{u_{1}-u_{2}}, \\
k=3,4,5, \ldots ; p \in \mathbb{P}, u_{i} \in \mathbb{C}: u_{i} \neq u_{j}, i \neq j .
\end{gathered}
$$

The divided differences which appear in the representation of the Newton interpolation polynomial, are defined recursively as (see $[\mathrm{G}],[\mathrm{BG}]$ ):

$$
\begin{gathered}
p\left(u_{0} ; u_{1} ; \ldots ; u_{k} ; u_{k+1}\right)=\frac{p\left(u_{1} ; u_{2} ; \ldots ; u_{k} ; u_{k+1}\right)-p\left(u_{0} ; u_{1} ; \ldots ; u_{k}\right)}{u_{k+1}-u_{0}}, \\
u_{i} \in \mathbb{C}: u_{i} \neq u_{j}, i \neq j ; k=0,1, \ldots
\end{gathered}
$$


This definition is equivalent to (2.3) because it is known that the divided differences are symmetric functions of their arguments.

The divided differences have the following properties (the proofs in [BZ] are for the case of real $u_{i}$ but for the case of complex $u_{i}$ the proofs are the same):

$$
\begin{gathered}
p\left(u_{1} ; u_{2} ; u_{3} ; \ldots ; u_{k}\right)=p\left(u_{2} ; u_{1} ; u_{3} ; \ldots ; u_{k}\right)=p\left(u_{3} ; u_{1} ; u_{2} ; \ldots ; u_{k}\right)=\ldots ; \\
\left(p_{1}+p_{2}\right)\left(u_{1} ; u_{2} ; u_{3} ; \ldots ; u_{k}\right)=p_{1}\left(u_{1} ; u_{2} ; u_{3} ; \ldots ; u_{k}\right)+p_{2}\left(u_{1} ; u_{2} ; u_{3} ; \ldots ; u_{k}\right), p_{1}, p_{2} \in \mathbb{P} ; \\
(\alpha p)\left(u_{1} ; u_{2} ; u_{3} ; \ldots ; u_{k}\right)=\alpha p\left(u_{1} ; u_{2} ; u_{3} ; \ldots ; u_{k}\right), \alpha \in \mathbb{C} .
\end{gathered}
$$

If $p(u)=u^{n}$ then

$$
p\left(u_{0} ; u_{1} ; u_{2} ; \ldots ; u_{k+1}\right)=\sum_{\beta_{0}+\beta_{1}+\ldots+\beta_{k+1}=n-k-1} u_{0}^{\beta_{0}} u_{1}^{\beta_{1}} \ldots u_{k+1}^{\beta_{k+1}}, k<n .
$$

If $p$ is a polynomial of $n$-th degree then

$$
p\left(u_{0} ; u_{1} ; u_{2} ; \ldots ; u_{n}\right)=\text { const and } p\left(u_{0} ; u_{1} ; u_{2} ; \ldots ; u_{n+j}\right)=0, j=1,2, \ldots
$$

For the divided differences the following formula holds:

$$
p\left(u_{0} ; u_{1} ; u_{2} ; \ldots ; u_{k}\right)=\sum_{j=0}^{k} \frac{p\left(u_{j}\right)}{\prod_{i \neq j}\left(u_{j}-u_{i}\right)} .
$$

Let us consider the following matrix:

$$
W=\left(\begin{array}{ccccc}
p_{0}(\lambda) & p_{0}(\lambda \varepsilon) & p_{0}\left(\lambda \varepsilon^{2}\right) & \ldots & p_{0}\left(\lambda \varepsilon^{N-1}\right) \\
p_{1}(\lambda) & p_{1}(\lambda \varepsilon) & p_{1}\left(\lambda \varepsilon^{2}\right) & \ldots & p_{1}\left(\lambda \varepsilon^{N-1}\right) \\
p_{2}(\lambda) & p_{2}(\lambda \varepsilon) & p_{2}\left(\lambda \varepsilon^{2}\right) & \ldots & p_{2}\left(\lambda \varepsilon^{N-1}\right) \\
\vdots & \vdots & \vdots & \ddots & \vdots \\
p_{N-1}(\lambda) & p_{N-1}(\lambda \varepsilon) & p_{N-1}\left(\lambda \varepsilon^{2}\right) & \ldots & p_{N-1}\left(\lambda \varepsilon^{N-1}\right)
\end{array}\right)
$$

where $\varepsilon$ is a primitive $\mathrm{N}$-th root of unity. Note that if $\lambda=0$ then the matrix $W$ is singular. Let us consider the case $\lambda \neq 0$. Subtract the first column from the others and after the subtraction divide the remainding columns by $\lambda \varepsilon^{k}-\lambda$ ( $k$ is the index of the column). We shall obtain a matrix

$$
W_{1}=\left(\begin{array}{ccccc}
p_{0}(\lambda) & \frac{p_{0}(\lambda \varepsilon)-p_{0}(\lambda)}{\lambda \varepsilon-\lambda} & \frac{p_{0}\left(\lambda \varepsilon^{2}\right)-p_{0}(\lambda)}{\lambda \varepsilon^{2}-\lambda} & \ldots & \frac{p_{0}\left(\lambda \varepsilon^{N-1}\right)-p_{0}(\lambda)}{\lambda \varepsilon^{N-1}-\lambda} \\
p_{1}(\lambda) & \frac{p_{1}(\lambda \varepsilon)-p_{1}(\lambda)}{\lambda \varepsilon-\lambda} & \frac{p_{1}\left(\lambda \varepsilon^{2}\right)-p_{1}(\lambda)}{\lambda \varepsilon^{2}-\lambda} & \ldots & \frac{p_{1}\left(\lambda \varepsilon^{N-1}\right)-p_{1}(\lambda)}{\lambda \varepsilon^{N-1}-\lambda} \\
\vdots & \vdots & \vdots & \ddots & \vdots \\
p_{N-1}(\lambda) & \frac{p_{N-1}(\lambda \varepsilon)-p_{N-1}(\lambda)}{\lambda \varepsilon-\lambda} & \frac{p_{N-1}\left(\lambda \varepsilon^{2}\right)-p_{N-1}(\lambda)}{\lambda \varepsilon^{2}-\lambda} & \ldots & \frac{p_{N-1}\left(\lambda \varepsilon^{N-1}\right)-p_{N-1}(\lambda)}{\lambda \varepsilon^{N-1}-\lambda}
\end{array}\right)=
$$




$$
=\left(\begin{array}{ccccc}
p_{0}(\lambda) & 0 & 0 & \ldots & 0 \\
p_{1}(\lambda) & p_{1}(\lambda \varepsilon ; \lambda) & p_{1}\left(\lambda \varepsilon^{2} ; \lambda\right) & \ldots & p_{1}\left(\lambda \varepsilon^{N-1} ; \lambda\right) \\
\vdots & \vdots & \vdots & \ddots & \vdots \\
p_{N-1}(\lambda) & p_{N-1}(\lambda \varepsilon ; \lambda) & p_{N-1}\left(\lambda \varepsilon^{2} ; \lambda\right) & \ldots & p_{N-1}\left(\lambda \varepsilon^{N-1} ; \lambda\right)
\end{array}\right)
$$

Now, subtracting from the $3,4, \ldots, \mathrm{N}-1$ column the second one and dividing by $\lambda \varepsilon^{k}-\lambda \varepsilon$ ( $k$ is the index of the column) we have

$$
\begin{gathered}
W_{2}=\left(\begin{array}{ccccc}
p_{0}(\lambda) & 0 & 0 & \ldots & 0 \\
p_{1}(\lambda) & p_{1}(\lambda \varepsilon ; \lambda) & \frac{p_{1}\left(\lambda \varepsilon^{2} ; \lambda\right)-p_{1}(\lambda \varepsilon ; \lambda)}{\lambda \varepsilon^{2}-\lambda \varepsilon} & \ldots & \frac{p_{1}\left(\lambda \varepsilon^{N-1} ; \lambda\right)-p_{1}(\lambda \varepsilon ; \lambda)}{\lambda \varepsilon^{N-1}-\lambda \varepsilon} \\
p_{2}(\lambda) & p_{2}(\lambda \varepsilon ; \lambda) & \frac{p_{2}\left(\lambda \varepsilon^{2} ; \lambda\right)-p_{2}(\lambda \varepsilon ; \lambda)}{\lambda \varepsilon^{2}-\lambda \varepsilon} & \ldots & \frac{p_{2}\left(\lambda \varepsilon^{N-1} ; \lambda\right)-p_{2}(\lambda \varepsilon ; \lambda)}{\lambda \varepsilon^{N-1}-\lambda \varepsilon} \\
\vdots & \vdots & \vdots & \ddots & \vdots \\
p_{N-1}(\lambda) & p_{N-1}(\lambda \varepsilon ; \lambda) & \frac{p_{N-1}\left(\lambda \varepsilon^{2} ; \lambda\right)-p_{N-1}(\lambda \varepsilon ; \lambda)}{\lambda \varepsilon^{2}-\lambda \varepsilon} & \ldots & \frac{p_{N-1}\left(\lambda \varepsilon^{N-1} ; \lambda\right)-p_{N-1}(\lambda \varepsilon ; \lambda)}{\lambda \varepsilon^{N-1}-\lambda \varepsilon}
\end{array}\right)= \\
=\left(\begin{array}{ccccc}
p_{0}(\lambda) & 0 & 0 & \ldots & \\
p_{1}(\lambda) & p_{1}(\lambda \varepsilon ; \lambda) & 0 & \ldots & 0 \\
p_{2}(\lambda) & p_{2}(\lambda \varepsilon ; \lambda) & p_{2}\left(\lambda \varepsilon^{2} ; \lambda \varepsilon ; \lambda\right) & \ldots & p_{2}\left(\lambda \varepsilon^{N-1} ; \lambda \varepsilon ; \lambda\right) \\
\vdots & \vdots & \vdots & \ddots & \vdots \\
p_{N-1}(\lambda) & p_{N-1}(\lambda \varepsilon ; \lambda) & p_{N-1}\left(\lambda \varepsilon^{2} ; \lambda \varepsilon ; \lambda\right) & \ldots & p_{N-1}\left(\lambda \varepsilon^{N-1} ; \lambda \varepsilon ; \lambda\right)
\end{array}\right) .
\end{gathered}
$$

In the (N-1)-th step we get

$$
W_{N-1}=\left(\begin{array}{ccccc}
p_{0}(\lambda) & 0 & 0 & \ldots & 0 \\
p_{1}(\lambda) & p_{1}(\lambda \varepsilon ; \lambda) & 0 & \ldots & 0 \\
p_{2}(\lambda) & p_{2}(\lambda \varepsilon ; \lambda) & p_{2}\left(\lambda \varepsilon^{2} ; \lambda \varepsilon ; \lambda\right) & \ldots & 0 \\
\vdots & \vdots & \vdots & \ddots & \vdots \\
p_{N-1}(\lambda) & p_{N-1}(\lambda \varepsilon ; \lambda) & p_{N-1}\left(\lambda \varepsilon^{2} ; \lambda \varepsilon ; \lambda\right) & \ldots & p_{N-1}\left(\lambda \varepsilon^{N-1} ; \lambda \varepsilon^{N-2} ; \ldots ; \lambda \varepsilon ; \lambda\right)
\end{array}\right) .
$$

From (2.4) and $(2.5)$ it follows that $p_{k}\left(\lambda \varepsilon^{k} ; \lambda \varepsilon^{k-1} ; \ldots ; \lambda\right)=\mu_{k}$, where $\mu_{k}$ is the leading coefficient of $p_{k}$. So,

$$
\operatorname{det} W_{N-1}=\prod_{k=0}^{N-1} \mu_{k} \neq 0
$$

for $\lambda \neq 0$.

Notice that for $\lambda=0$ we can also consider the matrix $W_{N-1}$. Really, the entries of $W_{N-1}$ are polynomials of $\lambda$ as follows from (2.4),(2.5), and we can 
take their limit values at $\lambda=0$. In the case $\lambda=0$ the relation $\operatorname{det} W_{N-1} \neq 0$ also holds true.

This observation give us an idea how to construct the basic solutions for all $\lambda \in \mathbb{C}$ from the vectors of polynomials $\left(p_{0}\left(\lambda \varepsilon^{k}\right), p_{1}\left(\lambda \varepsilon^{k}\right), \ldots\right)^{T}, k=$ $0,1, \ldots, N-1$.

Let us define the following vectors:

$$
\begin{aligned}
& \vec{p}(\lambda)=\left(\begin{array}{c}
p_{0}(\lambda) \\
p_{1}(\lambda) \\
\cdot \\
\cdot \\
\cdot
\end{array}\right), \vec{p}(\lambda \varepsilon ; \lambda)=\left(\begin{array}{c}
p_{0}(\lambda \varepsilon ; \lambda) \\
p_{1}(\lambda \varepsilon ; \lambda) \\
\cdot \\
\cdot \\
\cdot
\end{array}\right), \vec{p}\left(\lambda \varepsilon^{k} ; \lambda \varepsilon^{k-1} ; \ldots ; \lambda \varepsilon ; \lambda\right)= \\
& =\left(\begin{array}{c}
p_{0}\left(\lambda \varepsilon^{k} ; \ldots ; \lambda \varepsilon ; \lambda\right) \\
p_{1}\left(\lambda \varepsilon^{k} ; \ldots ; \lambda \varepsilon ; \lambda\right) \\
\cdot \\
\cdot \\
\cdot
\end{array}\right), k=2,3, \ldots, N-1 ; \text { with } \lambda \in \mathbb{C} \backslash\{0\} .
\end{aligned}
$$

Notice that these vectors are linear combinations of $\vec{p}(\lambda), \vec{p}(\lambda \varepsilon), \ldots, \vec{p}\left(\lambda \varepsilon^{N-1}\right)$ as follows from (2.7). They are solutions of (2.2) because $\vec{p}(\lambda), \vec{p}(\lambda \varepsilon), \ldots, \vec{p}\left(\lambda \varepsilon^{N-1}\right)$ are solutions. First $N$ rows of a matrix $\left(\vec{p}(\lambda), \vec{p}(\lambda \varepsilon ; \lambda), \ldots, \vec{p}\left(\lambda \varepsilon^{N-1}, \lambda \varepsilon^{N-2} ; \ldots ; \lambda\right)\right)$ coincide with $W_{N-1}$. So, $\vec{p}(\lambda), \vec{p}(\lambda \varepsilon ; \lambda), \ldots, \vec{p}\left(\lambda \varepsilon^{N-1}, \lambda \varepsilon^{N-2} ; \ldots ; \lambda\right)$ are $N$ linear independent solutions of $(2.2)$.

When $\lambda=0$ we write

$$
\begin{gathered}
\vec{p}(0 ; 0)=\left(\begin{array}{c}
\lim _{\lambda \rightarrow 0} p_{0}(\lambda \varepsilon ; \lambda) \\
\lim _{\lambda \rightarrow 0} p_{1}(\lambda \varepsilon ; \lambda) \\
\cdot \\
\cdot \\
\cdot
\end{array}\right), \vec{p}(0 ; 0 ; \ldots ; 0 ; 0)= \\
=\left(\begin{array}{c}
\lim _{\lambda \rightarrow 0} p_{0}\left(\lambda \varepsilon^{k} ; \ldots ; \lambda \varepsilon ; \lambda\right) \\
\lim _{\lambda \rightarrow 0} p_{1}\left(\lambda \varepsilon^{k} ; \ldots ; \lambda \varepsilon ; \lambda\right) \\
\cdot \\
\cdot
\end{array}\right), k=2,3, \ldots, N-1 .
\end{gathered}
$$

The limits in (2.10) exist because of (2.5) and the vectors in (2.10) are solutions of (2.2). It is not hard to see that they are linearly independent. 
Now we shall construct another version of the first N solutions of (2.1). Consider the following vectors:

$$
\begin{aligned}
& R_{m, N}^{N}(\vec{p})(\lambda)=\left(\begin{array}{c}
R_{m, N}\left(p_{0}\right)(\lambda) \\
R_{m, N}\left(p_{1}\right)(\lambda) \\
\vdots \\
R_{m, N}\left(p_{N-1}\right)(\lambda)
\end{array}\right), R_{m, N}(\vec{p})(\lambda)=\left(\begin{array}{c}
R_{m, N}\left(p_{0}\right)(\lambda) \\
R_{m, N}\left(p_{1}\right)(\lambda) \\
\vdots \\
R_{m, N}\left(p_{N-1}\right)(\lambda) \\
\vdots
\end{array}\right) \\
& m=0,1, \ldots, N-1,
\end{aligned}
$$

where $R_{m, N}(p)(\lambda)=\sum_{j} a_{N j+m} \lambda^{N j}$ if $p(\lambda)=\sum_{i} a_{i} \lambda^{i}$ [D1, p. 90]. The following is true [D1, p. 90]:

$$
R_{m, N}(p)(\lambda)=\frac{1}{N \lambda^{m}} \sum_{k=0}^{N-1} \varepsilon^{-m k} p\left(\lambda \varepsilon^{k}\right), \lambda \neq 0 .
$$

If $m \leq \operatorname{deg}(p(\lambda))<N$ then $R_{m, N}(p)(\lambda)=a_{m}$ and if $\operatorname{deg}(p(\lambda))<m$ then $R_{m, N}(p)(\lambda)=0$. From this we conclude that the matrix $V=\left(R_{0, N}^{N}(\vec{p})(\lambda)\right.$, $\left.R_{1, N}^{N}(\vec{p})(\lambda), \ldots, R_{N-1, N}^{N}(\vec{p})(\lambda)\right)$ is lower-triangular and non-singular. As follows from (2.11) the vectors $R_{m, N}^{N}(\vec{p})(\lambda), m=0,1, \ldots, N-1$ are linear combinations of solutions of (2.2) for $\lambda \neq 0$. So, they are also solutions and they are linearly independent because $\operatorname{det} V \neq 0$. If $\lambda=0$ then we can consider the limit vectors when $\lambda \rightarrow 0$. These vectors will be linearly independent.

Now we turn to the construction of another set of $\mathrm{N}$ solutions of (2.1). Define a bilinear functional $\sigma(u, v)$ in $\mathbb{P}$ :

$$
\sigma(u, v)=\sum_{i} a_{i} \overline{b_{i}} \text { with } u=\sum_{i} a_{i} p_{i}(\lambda), v=\sum_{i} b_{i} p_{i}(\lambda) .
$$

This functional satisfies:

$$
\begin{gathered}
\sigma\left(p_{i}, p_{j}\right)=\delta_{i j}, i, j=0,1,2, \ldots ; \\
\sigma\left(\lambda^{N} u_{1}(\lambda), u_{2}(\lambda)\right)=\sigma\left(u_{1}(\lambda), \lambda^{N} u_{2}(\lambda)\right), u_{1}, u_{2} \in \mathbb{P} .
\end{gathered}
$$

In other words $\lambda^{N}: \mathbb{P} \rightarrow \mathbb{P}$ is a symmetric operator with respect to $\sigma$.

Consider the following polynomials:

$$
F_{m, l, j, k}=\sigma_{u}\left(u^{l} \frac{R_{m, N}\left(p_{k}\right)(u)-R_{m, N}\left(p_{k}\right)(\lambda)}{u^{N}-\lambda^{N}}, p_{j}(u)\right), k=0,1,2, \ldots ;
$$




$$
m, l, j=0,1, \ldots, N-1 .
$$

Substituting $F_{m, l, j, k}(\lambda)$ in the left-hand side of (2.1) instead of $y_{k}, k=0,1,2, \ldots$, and using the fact that $R_{m, N}\left(p_{k}\right)(u), R_{m, N}\left(p_{k}\right)(\lambda)$ satisfy $(2.1)$ with the parameters $u$ and $\lambda$ respectively, we get:

$$
\begin{gathered}
\sum_{i=1}^{N}\left(\overline{\alpha_{k-i, i}} F_{m, l, j, k-i}(\lambda)+\alpha_{k, i} F_{m, l, j, k+i}(\lambda)\right)+\alpha_{k, 0} F_{m, l, j, k}(\lambda)= \\
=\sigma_{u}\left(u^{l} \frac{u^{N} R_{m, N}\left(p_{k}\right)(u)-\lambda^{N} R_{m, N}\left(p_{k}\right)(\lambda)}{u^{N}-\lambda^{N}}, p_{j}(u)\right)= \\
=\sigma_{u}\left(u^{l} \frac{u^{N} R_{m, N}\left(p_{k}\right)(u)-\lambda^{N} R_{m, N}\left(p_{k}\right)(u)+\lambda^{N} R_{m, N}\left(p_{k}\right)(u)-\lambda^{N} R_{m, N}\left(p_{k}\right)(\lambda)}{u^{N}-\lambda^{N}},\right. \\
\left.p_{j}(u)\right)=\sigma_{u}\left(u^{l}\left(R_{m, N}\left(p_{k}\right)(u)+\lambda^{N} \frac{R_{m, N}\left(p_{k}\right)(u)-R_{m, N}\left(p_{k}\right)(\lambda)}{u^{N}-\lambda^{N}}\right), p_{j}(u)\right)= \\
=\sigma_{u}\left(u^{l} R_{m, N}\left(p_{k}\right)(u), p_{j}(u)\right)+\lambda^{N} F_{m, l, j, k}(\lambda), k=0,1, \ldots ; m, l, j=0,1, \ldots, N-1 .
\end{gathered}
$$

Notice that

$$
\sum_{m=0}^{N-1} u^{m} R_{m, N}\left(p_{k}\right)(u)=p_{k}(u) .
$$

Denote

$$
F_{j, k}=\sum_{m=0}^{N-1} F_{m, m, j, k}(\lambda), k=0,1, \ldots ; j=0,1, \ldots, N-1
$$

Then

$$
\begin{gathered}
\sum_{m=0}^{N-1}\left[\sum_{i=1}^{N}\left(\overline{\alpha_{k-i, i}} F_{m, m, j, k-i}(\lambda)+\alpha_{k, i} F_{m, m, j, k+i}(\lambda)\right)+\alpha_{k, 0} F_{m, m, j, k}(\lambda)\right]= \\
=\sigma\left(p_{k}(u), p_{j}(u)\right)+\lambda^{N} F_{j, k}(\lambda) . \\
\sum_{i=1}^{N}\left(\overline{\alpha_{k-i, i}} F_{j, k-i}(\lambda)+\alpha_{k, i} F_{j, k+i}(\lambda)\right)+\alpha_{k, 0} F_{j, k}(\lambda)= \\
=\sigma\left(p_{k}(u), p_{j}(u)\right)+\lambda^{N} F_{m, l, j, k}(\lambda) .
\end{gathered}
$$


Let us take arbitrary $j=0,1,2, \ldots, N-1$. Then for $k=j+1, j+2, \ldots, N, N+$ $1, N+2, \ldots$, the equation of the type (2.1) with $F_{j, k}$ is fulfilled. So the vectors $\vec{F}_{j}(\lambda)=\left(\begin{array}{c}F_{j, 0}(\lambda) \\ F_{j, 1}(\lambda) \\ \vdots \\ F_{j, N-1}(\lambda) \\ \vdots\end{array}\right), j=0,1, \ldots, N-1$, are solutions of $(2.1)$.

From (2.15) it is clear that for $k=0 \vec{F}_{0}(\lambda)$ does not satisfy (2.1). So, it does not lie in the

$$
\operatorname{span}\left[\vec{p}(\lambda), \vec{p}(\lambda \varepsilon ; \lambda), \ldots, \vec{p}\left(\lambda \varepsilon^{N-1}, \ldots, \lambda \varepsilon ; \lambda\right)\right]
$$

(or in the $\operatorname{span}\left[R_{0, N}(\vec{p})(\lambda), R_{1, N}(\vec{p})(\lambda), \ldots, R_{N-1, N}(\vec{p})(\lambda)\right]$ ).

For $k=1$ we can see that $\vec{F}_{1}(\lambda)$ does not satisfy $(2.1)$. Then it does not lie in the

$$
\operatorname{span}\left[\vec{p}(\lambda), \vec{p}(\lambda \varepsilon ; \lambda), \ldots, \vec{p}\left(\lambda \varepsilon^{N-1}, \ldots, \lambda \varepsilon ; \lambda\right) ; \vec{F}_{0}(\lambda)\right]
$$

(or in the $\left.\operatorname{span}\left[R_{0, N}(\vec{p})(\lambda), R_{1, N}(\vec{p})(\lambda), \ldots, R_{N-1, N}(\vec{p})(\lambda) ; \vec{F}_{0}(\lambda)\right]\right)$.

We can continue this consideration and obtain that $\vec{F}_{k}(\lambda)$ does not lie in the

$$
\operatorname{span}\left[\vec{p}(\lambda), \vec{p}(\lambda \varepsilon ; \lambda), \ldots, \vec{p}\left(\lambda \varepsilon^{N-1}, \ldots, \lambda \varepsilon ; \lambda\right) ; \vec{F}_{0}(\lambda), \vec{F}_{1}(\lambda), \ldots, \vec{F}_{k-1}(\lambda)\right]
$$

(or in the $\left.\operatorname{span}\left[R_{0, N}(\vec{p})(\lambda), R_{1, N}(\vec{p})(\lambda), \ldots, R_{N-1, N}(\vec{p})(\lambda) ; \vec{F}_{0}(\lambda), \vec{F}_{1}(\lambda), \ldots, \vec{F}_{k-1}(\lambda)\right]\right)$, $k=2,3, \ldots N-1$.

So the vectors

$$
\vec{p}(\lambda), \vec{p}(\lambda \varepsilon ; \lambda), \ldots, \vec{p}\left(\lambda \varepsilon^{N-1}, \ldots, \lambda \varepsilon ; \lambda\right) ; \vec{F}_{0}(\lambda), \vec{F}_{1}(\lambda), \ldots, \vec{F}_{N-1}(\lambda),
$$

or the vectors

$$
R_{0, N}(\vec{p})(\lambda), R_{1, N}(\vec{p})(\lambda), \ldots, R_{N-1, N}(\vec{p})(\lambda) ; \vec{F}_{0}(\lambda), \vec{F}_{1}(\lambda), \ldots, \vec{F}_{N-1}(\lambda),
$$

are $2 N$ linearly independent solutions of (2.1).

\section{$\S 3$. Integral representations for basic solutions.}

Now we are going to present different integral representations of the basic solutions constructed in section 2. For this aim we shall use extensions of Favard's theorem. 
Let us consider again $(2 N)$-order linear difference equation $(2.1)\left(2.1^{\prime}\right)$ and the set of polynomials $\left\{p_{n}(\lambda)\right\}_{n=0}^{\infty}$ satisfying (2.2). For the functional $\sigma(u, v)$ defined as in (12), using Duran's result [D2, Theorem 3.1, p. 93] we have the representation (for real coefficients in (2.1)):

$$
\begin{gathered}
\sigma(u, v)=\sum_{m, m^{\prime}=1}^{N} \int_{R} R_{N, m-1}(u)(x) \overline{R_{N, m^{\prime}-1}(v)(x)} d \mu_{m, m^{\prime}}= \\
=\int_{R}\left(R_{N, 0}(u)(x), R_{N, 1}(u)(x), \ldots, R_{N, N-1}(u)(x)\right) d \mu(x)\left(\begin{array}{c}
R_{N, 0}(v)(x) \\
R_{N, 1}(v)(x) \\
\ldots \\
R_{N, N-1}(v)(x)
\end{array}\right),
\end{gathered}
$$

where $\mu(x)=\left(\mu_{m, m^{\prime}}(x)\right)_{m, m^{\prime}=1}^{N}$ is a positive-definite $(N * N)$ matrix of measures, $R_{N, m}(u)(x)=\sum_{n} \frac{u^{(n N+m)}(0)}{(n N+m) !} x^{N}$ (Notice that $R_{N, m}(u)(x) \neq R_{m, N}(u)(x)$ so the order of the indexes is important).

Such a representation was first obtained by Duran for non-positive matrix of measures $\mu$.

Then, using results in [Z4], [Z5] we can write the following representations:

$$
\sigma(u, v)=\int_{P_{N}}\left(u(\lambda), u(\lambda \varepsilon), u\left(\lambda \varepsilon^{2}\right), \ldots, u\left(\lambda \varepsilon^{N-1}\right) J_{\lambda} d \sigma(\lambda) J_{\lambda}^{*}\left(\begin{array}{c}
v(\lambda) \\
v(\lambda \varepsilon) \\
\cdot \\
\cdot \\
v\left(\lambda \varepsilon^{N-1}\right)
\end{array}\right)\right.
$$

where $\varepsilon$ is a $\mathrm{N}$-th primitive root of unity, $J_{\lambda}=\left(a_{i, j}\right)_{i, j=1}^{N}, a_{i, j}=\frac{1}{\varepsilon^{i j} \lambda^{j}}, P_{N}=$ $\left\{\lambda \in \mathbb{C}: \lambda^{N} \in \mathbb{R}\right\} ; \sigma(\lambda)$ is a non-decreasing matrix-valued function on $P_{N}$ (i.e. on each of $2 N$ rays in $\left.P_{N}\right): \sigma(0)=0$. Notice that when $\lambda=0$ one must take the limit values as $\lambda \rightarrow 0$ of $\left(u(\lambda), u(\lambda \varepsilon), \ldots, u\left(\lambda \varepsilon^{N-1}\right) J_{\lambda}\right.$ and 


$$
\begin{aligned}
& \overline{J_{\lambda}^{*}\left(\begin{array}{c}
v(\lambda) \\
v(\lambda \varepsilon) \\
\cdot \\
v\left(\lambda \varepsilon^{N-1}\right)
\end{array}\right)} \text { under the integral; }
\end{aligned}
$$

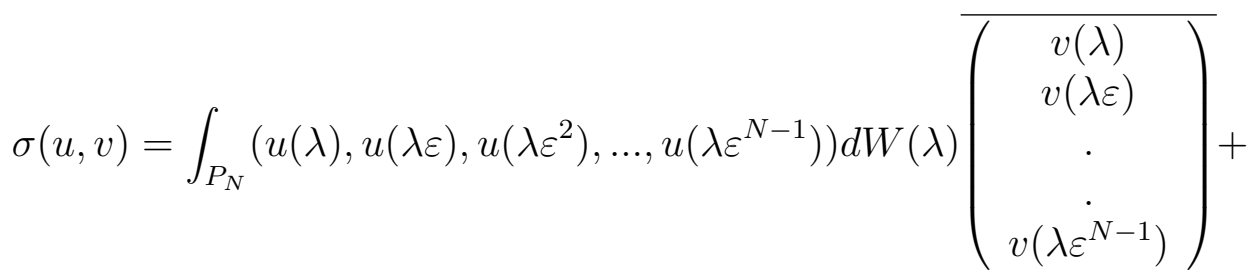

$$
\begin{aligned}
& +\left(u(0), u^{\prime}(0), u^{\prime \prime}(0), \ldots, u^{(N-1)}(0)\right) M\left(\begin{array}{c}
v(0) \\
v^{\prime}(0) \\
\cdot \\
\cdot \\
v^{(N-1)}(0)
\end{array}\right),
\end{aligned}
$$

where $W(\lambda)$ is a non-decreasing matrix-valued function on $P_{N} \backslash\{0\}$. With the integral at $\lambda=0$ we understand the improper integral. Here $M \geq 0$ is a $(N \times N)$ complex numerical matrix.

By the integrals over $P_{N}$ we understand a sum of integrals over each ray in $P_{N}$.

The method of proof for (3.2)(3.3) (step by step the construction of a spectral measure) differs from the method of proof for (3.1) (using operator theory tools). It was used for construction of examples of measures ([Z1], [Z6]). From (3.2), (3.3) it is obvious that $\sigma\left(\lambda^{N} u, v\right)=\sigma\left(u, \lambda^{N} v\right)$. From (3.3) it was shown in [Z5] how different families of polynomials are included in the class of polynomials from (3.3): orthonormal polynomials on $\mathbb{R}$, orthonormal polynomials on rays with a scalar measure studied by Milovanović [Mil] and in [MS], Sobolev type orthonormal polynomials with discrete measure at zero.

For polynomials satisfying (2.2) a connection with matrix orthogonal polynomials on $\mathbb{R}$ was studied in $[\mathrm{DA}],[\mathrm{Z} 5]$. But the matrix orthonormality relation written in terms of polynomials $\left\{p_{n}(\lambda)\right\}_{n=0}^{\infty}$ does not give a functional $\sigma(u, v)$ such that $\sigma\left(p_{i}, p_{j}\right)=\delta_{i, j}$. So we shall not consider it here. But we would like to present here a remark which seems to be important concerning the Favard theorem for matrix polynomials: 
Remark. Duran, Van Assche [DA, p. 263], [D3, p. 66] assert that the Favard theorem for matrix polynomials was established by Aptekarev and Nikishin in 1982 in [AN] (Also Lopez-Rodriguez refers to this paper in [L, p. 248]). But Aptekarev and Nikishin wrote [AN, p. 328] that the Jacobi matrix $L$ with matrix entries $\left\{V_{J}, E_{j}\right\}_{j=0}^{\infty}: V_{j}^{*}=V_{j}$, det $E_{j} \neq 0$ always define in $l_{2}\left(\mathbb{C}^{N}\right)$ a self-adjoint operator. This is not true even for numerical Jacobi matrices. Moreover, the Favard's theorem for operator-valued polynomials was established by Berezanskiy [B, Chapter 7, §2, Theorem 2.4, p. 571] in 1965, and in particular it holds true for matrix-valued polynomials. So it is correct to refer to $[\mathrm{B}]$ in this question.

$$
\text { Let us write representations for } \vec{F}_{j}(\lambda)=\left(\begin{array}{c}
F_{j, 0}(\lambda) \\
F_{j, 1}(\lambda) \\
\vdots \\
F_{j, N-1}(\lambda) \\
\vdots
\end{array}\right), j=0,1, \ldots, N-
$$

1, which are the $N$ solutions of (2.1). Using the definitions of $F_{j, k}$ and $F_{m, l, j, k}$ we have:

$$
\begin{gathered}
F_{j, k}(z)=\sum_{m=0}^{N-1} F_{m, m, j, k}(z)=\sum_{m=0}^{N-1} \sigma_{u}\left(u^{m} \frac{R_{m, N}\left(p_{k}\right)(u)-R_{m, N}\left(p_{k}\right)(z)}{u^{N}-z^{N}}, p_{j}(u)\right)= \\
=\sigma_{u}\left(\frac{p_{k}(u)-\sum_{m=0}^{N-1} u^{m} R_{m, N}\left(p_{k}\right)(z)}{u^{N}-z^{N}}, p_{j}(u)\right) \\
\text { with } j=0,1, \ldots, N-1 ; k=0,1, \ldots
\end{gathered}
$$

Then using (3.1)(3.2)(3.3) we have:

$$
\begin{gathered}
F_{j, k}(z)=\int_{R}\left(R_{N, 0 ; y}\left(\frac{p_{k}(y)-\sum_{m=0}^{N-1} y^{m} R_{m, N}\left(p_{k}\right)(z)}{y^{N}-z^{N}}\right)(x),\right. \\
R_{N, 1 ; y}\left(\frac{p_{k}(y)-\sum_{m=0}^{N-1} y^{m} R_{m, N}\left(p_{k}\right)(z)}{y^{N}-z^{N}}\right)(x), \ldots, \\
\left.R_{N, N-1 ; y}\left(\frac{p_{k}(y)-\sum_{m=0}^{N-1} y^{m} R_{m, N}\left(p_{k}\right)(z)}{y^{N}-z^{N}}\right)(x)\right) d \mu(x)\left(\begin{array}{c}
R_{N, 0}\left(p_{j}\right)(x) \\
R_{N, 1}\left(p_{j}\right)(x) \\
\ldots \\
R_{N, N-1}\left(p_{j}\right)(x)
\end{array}\right),
\end{gathered}
$$


where $R_{N, m ; y}(R)(x)$ means $R_{N, m}(R)(x)$ which acts on $R$ as on a function of $y$;

$$
\begin{aligned}
& \int_{P_{N}}\left(\frac{p_{k}(\lambda)-\sum_{m=0}^{N-1} \lambda^{m} R_{m, N}\left(p_{k}\right)(z)}{\lambda^{N}-z^{N}}, \frac{p_{k}(\lambda \varepsilon)-\sum_{m=0}^{N-1}(\lambda \varepsilon)^{m} R_{m, N}\left(p_{k}\right)(z)}{(\lambda \varepsilon)^{N}-z^{N}}, \ldots,\right. \\
& \frac{p_{k}\left(\lambda \varepsilon^{N-1}\right)-\sum_{m=0}^{N-1}\left(\lambda \varepsilon^{N-1}\right)^{m} R_{m, N}\left(p_{k}\right)(z)}{\left(\lambda \varepsilon^{N-1}\right)^{N}-z^{N}} J_{\lambda} d \sigma(\lambda) J_{\lambda}^{*} \overline{\left(\begin{array}{c}
p_{j}(\lambda) \\
p_{j}(\lambda \varepsilon) \\
\cdot \\
\cdot \\
p_{j}\left(\lambda \varepsilon^{N-1}\right)
\end{array}\right)}, \\
& \int_{P_{N}}\left(\frac{p_{k}(\lambda)-\sum_{m=0}^{N-1} \lambda^{m} R_{m, N}\left(p_{k}\right)(z)}{\lambda^{N}-z^{N}}, \frac{p_{k}(\lambda \varepsilon)-\sum_{m=0}^{N-1}(\lambda \varepsilon)^{m} R_{m, N}\left(p_{k}\right)(z)}{(\lambda \varepsilon)^{N}-z^{N}}, \ldots\right. \\
& \frac{p_{k}\left(\lambda \varepsilon^{N-1}\right)-\sum_{m=0}^{N-1}\left(\lambda \varepsilon^{N-1}\right)^{m} R_{m, N}\left(p_{k}\right)(z)}{\left(\lambda \varepsilon^{N-1}\right)^{N}-z^{N}} d W(\lambda)\left(\begin{array}{c}
p_{j}(\lambda) \\
p_{j}(\lambda \varepsilon) \\
\cdot \\
\cdot \\
p_{j}\left(\lambda \varepsilon^{N-1}\right)
\end{array}\right)+ \\
& +\left(\left.\frac{p_{k}(\lambda)-\sum_{m=0}^{N-1} \lambda^{m} R_{m, N}\left(p_{k}\right)(z)}{\lambda^{N}-z^{N}}\right|_{\lambda=0},\left.\left(\frac{p_{k}(\lambda)-\sum_{m=0}^{N-1} \lambda^{m} R_{m, N}\left(p_{k}\right)(z)}{\lambda^{N}-z^{N}}\right)_{\lambda}^{\prime}\right|_{\lambda=0}, \ldots,\right. \\
& \left.\left.\left(\frac{p_{k}(\lambda)-\sum_{m=0}^{N-1} \lambda^{m} R_{m, N}\left(p_{k}\right)(z)}{\lambda^{N}-z^{N}}\right)_{\lambda}^{(N-1)}\right|_{\lambda=0}\right) M\left(\begin{array}{c}
p_{j}(0) \\
p_{j}^{\prime}(0) \\
\cdot \\
\cdot \\
p_{j}^{(N-1)}(0)
\end{array}\right) .
\end{aligned}
$$

If $z \notin P_{N}$ then $x-z^{N} \neq 0, x \in \mathbb{R}$, as well as $\lambda^{N}-z^{N} \neq 0, \lambda \in P_{N}$.

Notice that

$$
\begin{gathered}
R_{N, m}(u)(x)=R_{m, N}(u)(\sqrt[N]{x})=\frac{1}{N(\sqrt[N]{x})^{m}} \sum_{k=0}^{N-1} \varepsilon^{-m k} u\left(\sqrt[N]{x} \varepsilon^{k}\right) \\
R_{N, m ; y}\left(\frac{p_{k}(y)-\sum_{i=0}^{N-1} y^{i} R_{i, N}\left(p_{k}\right)(z)}{y^{N}-z^{N}}\right)(x)= \\
=\frac{1}{N(\sqrt[N]{x})^{m}} \sum_{l=0}^{N-1} \varepsilon^{-m l} \frac{p_{k}\left(\sqrt[N]{x} \varepsilon^{l}\right)-\sum_{i=0}^{N-1}\left(\sqrt[N]{x} \varepsilon^{l}\right)^{i} R_{i, N}\left(p_{k}\right)(z)}{\left(\sqrt[N]{x} \varepsilon^{l}\right)^{N}-z^{N}}=
\end{gathered}
$$




$$
\begin{gathered}
=\frac{1}{x-z^{N}} \frac{1}{N(\sqrt[N]{x})^{m}} \sum_{l=0}^{N-1} \varepsilon^{-m l}\left(p_{k}\left(\sqrt[N]{x} \varepsilon^{l}\right)-\sum_{i=0}^{N-1}\left(\sqrt[N]{x} \varepsilon^{l}\right)^{i} R_{i, N}\left(p_{k}\right)(z)\right)= \\
=\frac{1}{x-z^{N}} R_{m, N ; y}\left(p_{k}(y)-\sum_{i=0}^{N-1} y^{i} R_{i, N}\left(p_{k}\right)(z)\right)(\sqrt[N]{x})= \\
=\frac{1}{x-z^{N}} R_{N, m ; y}\left(p_{k}(y)-\sum_{i=0}^{N-1} y^{i} R_{i, N}\left(p_{k}\right)(z)\right)(x) .
\end{gathered}
$$

Then

$$
\begin{aligned}
& F_{j, k}(z)=\int_{R} \frac{1}{x-z^{N}}\left(R_{N, 0 ; y}\left(p_{k}(y)-\sum_{m=0}^{N-1} y^{m} R_{m, N}\left(p_{k}\right)(z)\right)(x), R_{N, 1 ; y}\left(p_{k}(y)-\right.\right. \\
& \left.\left.-\sum_{m=0}^{N-1} y^{m} R_{m, N}\left(p_{k}\right)(z)\right)(x), \ldots, R_{N, N-1 ; y}\left(p_{k}(y)-\sum_{m=0}^{N-1} y^{m} R_{m, N}\left(p_{k}\right)(z)\right)(x)\right) * \\
& * d \mu(x)\left(\begin{array}{c}
R_{N, 0}\left(p_{j}\right)(x) \\
R_{N, 1}\left(p_{j}\right)(x) \\
\ldots \\
R_{N, N-1}\left(p_{j}\right)(x)
\end{array}\right), \\
& F_{j, k}(z)=\int_{P_{N}} \frac{1}{\lambda^{N}-z^{N}}\left(\left(p_{k}(\lambda)-\sum_{m=0}^{N-1} \lambda^{m} R_{m, N}\left(p_{k}\right)(z), p_{k}(\lambda \varepsilon)-\right.\right. \\
& \left.-\sum_{m=0}^{N-1}(\lambda \varepsilon)^{m} R_{m, N}\left(p_{k}\right)(z), \ldots, p_{k}\left(\lambda \varepsilon^{N-1}\right)-\sum_{m=0}^{N-1}\left(\lambda \varepsilon^{N-1}\right)^{m} R_{m, N}\left(p_{k}\right)(z)\right) J_{\lambda} * \\
& * d \sigma(\lambda) J_{\lambda}^{*}\left(\begin{array}{c}
p_{j}(\lambda) \\
p_{j}(\lambda \varepsilon) \\
\cdot \\
\cdot \\
p_{j}\left(\lambda \varepsilon^{N-1}\right)
\end{array}\right), \\
& F_{j, k}(z)=\int_{P_{N}} \frac{1}{\lambda^{N}-z^{N}}\left(p_{k}(\lambda)-\sum_{m=0}^{N-1} \lambda^{m} R_{m, N}\left(p_{k}\right)(z), p_{k}(\lambda \varepsilon)-\right. \\
& \left.-\sum_{m=0}^{N-1}(\lambda \varepsilon)^{m} R_{m, N}\left(p_{k}\right)(z), \ldots, p_{k}\left(\lambda \varepsilon^{N-1}\right)-\sum_{m=0}^{N-1}\left(\lambda \varepsilon^{N-1}\right)^{m} R_{m, N}\left(p_{k}\right)(z)\right) *
\end{aligned}
$$




$$
\begin{gathered}
* d W(\lambda)\left(\begin{array}{c}
p_{j}(\lambda) \\
p_{j}(\lambda \varepsilon) \\
\cdot \\
\cdot \\
p_{j}\left(\lambda \varepsilon^{N-1}\right)
\end{array}\right)+\left(\left.\frac{p_{k}(\lambda)-\sum_{m=0}^{N-1} \lambda^{m} R_{m, N}\left(p_{k}\right)(z)}{\lambda^{N}-z^{N}}\right|_{\lambda=0},\right. \\
\left.\left(\frac{p_{k}(\lambda)-\sum_{m=0}^{N-1} \lambda^{m} R_{m, N}\left(p_{k}\right)(z)}{\lambda^{N}-z^{N}}\right)_{\lambda}^{\prime}\right|_{\lambda=0}, \ldots, \\
\left.\left.\left(\frac{p_{k}(\lambda)-\sum_{m=0}^{N-1} \lambda^{m} R_{m, N}\left(p_{k}\right)(z)}{\lambda^{N}-z^{N}}\right)_{\lambda}^{(N-1)}\right|_{\lambda=0}\right) M\left(\begin{array}{c}
p_{j}(0) \\
p_{j}^{\prime}(0) \\
\cdot \\
\cdot \\
p_{j}^{(N-1)}(0)
\end{array}\right) \\
z \notin P_{N}, j=0,1, \ldots, N-1 ; k=0,1, \ldots .
\end{gathered}
$$

So, formulas (3.8)(3.9)(3.10) give us integral representations for polynomials $F_{j, k}(z)$ which are $N$ basic solutions of $(2.1)$.

Acknowledgements. The research of the first author has been supported by Dirección General de Investigación (Ministerio de Educación y Ciencia) of Spain under grant BFM 2003-06335-C03-02, and INTAS Research Network NeCCA INTAS 03-51-6637.

\section{List of references.}

[A] N.I. Akhiezer, The classical moments problem, Oliver and Boyd, Edinburgh, 1965.

[AN] A.I. Aptekarev, E.M. Nikishin, The scattering problem for a discrete Sturm-Liouville operator, Mat. Sb. 121(163) 3(7): 327-358 (1983) (Russian). [B] Yu. M. Berezanskiy, Expansions in Eigenfunctions of self-adjoint operators, Kiev, "Naukova dumka", 1965 (Russian).

[BZ] I.S. Berezin, N.P. Zhydkov, Methods of calculations, Vol. 1, Moscow, "Nauka", 1966 (Russian).

[C] T.S. Chihara, An Introduction to Orthogonal Polynomials, Gordon and Breach, New York, 1978. 
[D1] A.J. Duran, A Generalization of Favard's Theorem for Polynomials Satisfying a Recurrence Relation, J. of Approx. Th. 74, (1993), 83-109.

[D2] A.J. Duran, On orthogonal polynomials with respect to a positive definite matrix of measures, Canad. J. Math. 47(1), (1995), 88-112.

[D3] A.J. Duran, On orthogonal matrix polynomials, Proceedings of the International Workshop on Orthogonal Polynomials, M. Alfaro, R. ÁlvarezNodarse, G. López Lagomasino, and F. Marcellán Editors, Universidad Carlos III de Madrid, Leganés, 24-26 June, 1996, 63-72.

[DA] A.J. Duran and W. Van Assche, Orthogonal Matrix Polynomials and Higher Order Recurrence Relation, Linear Alg. and its Appl. 219: 261-280 (1995).

[G] A.O. Gelfond, Calculation of finite differences, Moscow, "Nauka", 1967 (Russian).

[L] P. Lopez-Rodriguez, The Nevanlinna parametrization for a matrix moment problem, Math. Scand. 89 (2001), 245-267.

[MA] F. Marcellán, R. Álvarez-Nodarse, On the "Favard theorem" and its extensions, J. of Comput. and Appl. Math. 127 (2001) 231-254.

[MS] F. Marcellán, G. Sansigre, On a Class of Matrix Orthogonal Polynomials on the Real Line, Linear Alg. and its Appl. 181: (1993) 97-109.

[MSz] F. Marcellán, F.H. Szafraniec, Integral Representations on Equipotential Curves and Harmonic Sets, Bull. Belg. Math Soc. Simon Stevin 11(2004) 457-468.

[Mil] G.V. Milovanović, A Class of Orthogonal Polynomials on the Radial Rays in the Complex Plane, J. Math. Anal. Appl. 206 (1997), 121-139.

[Z1] S. Zagorodniuk, On a five-diagonal Jacobi matrices and orthogonal polynomials on rays in the complex plane, Serdica Math. J., 24(3-4), (1998), 257-282.

[Z2] S.M. Zagorodniuk, Analog of Favard's theorem for polynomials connected with difference equation of 4-th order, Serdica Math. J., 27, (2001), 193-202.

[Z3] S.M. Zagorodnyuk, On a characteristic property of polynomials satisfying difference equation of 4-th order, Visnyk Kharkivs'kogo Univ., seriya "Matem., prykl. matem. i mech.", 2003 (Russian).

[Z4] S.M. Zagorodnyuk, On generalized Jacobi matrices and orthogonal polynomials, Abstracts for the Internat. Confer. "Inverse problems and Nonlinear Equat.", Kharkiv, 12-16 Aug. 2002, pp. 93-94. 
[Z5] S.M. Zagorodnyuk, On generalized Jacobi matrices and orthogonal polynomials, manuscript.

[Z6] S.M. Zagorodnyuk, Orthogonal polynomials on the real and the imaginary axes in the complex plane, Math. phyzika, analiz, geom., T. 9(3), (2002), 502-508 (Russian). 